\title{
Magnetic resonance imaging of solid urethral and peri-urethral lesions
}

\author{
Andrew Del Gaizo • Alvin C. Silva • Dora M. Lam-Himlin • \\ Brian C. Allen • John Leyendecker • Akira Kawashima
}

Received: 26 March 2013 /Revised: 25 April 2013 /Accepted: 29 April 2013 /Published online: 19 May 2013

(C) The Author(s) 2013. This article is published with open access at Springerlink.com

\begin{abstract}
Solid urethral and peri-urethral lesions are rare and encompass benign and malignant aetiologies. A diagnosis without imaging is often challenging secondary to non-specific clinical symptoms and overlapping findings at the time of physical examination. Magnetic resonance (MR) imaging may be helpful in confirming a diagnosis while providing anatomical detail and delineating disease extent. This article reviews the normal MR anatomy of the male and female urethra, the MR appearance of solid primary and secondary urethral lesions, and the MR appearance of solid urethral lesion mimics.

Teaching points

- MRI is an important imaging technique in the evaluation of the spectrum of solid urethral lesions.

- With excellent soft tissue resolution, MR is accurate in staging primary urethral carcinoma.

- Disruption of the zonal anatomy of the female urethral wall indicates peri-urethral extension.

- Be aware of benign urethral lesions, particularly those that may mimic solid urethral masses.
\end{abstract}

Keywords Urethra $\cdot$ Carcinoma $\cdot$ Magnetic resonance . Solid lesions

This article is based on Exhibit LL-URE2534 at the RSNA 2011 Annual Meeting, where it received the Certificate of Merit award

A. Del Gaizo $(\triangle) \cdot$ B. C. Allen · J. Leyendecker Department of Radiology, Wake Forest University, Medical Center Blvd., Winston-Salem, NC 27157, USA

e-mail: adelgaiz@wfubmc.edu

A. Del Gaizo • A. C. Silva

Department of Radiology, Mayo Clinic, Scottsdale, AZ, USA

D. M. Lam-Himlin

Department of Pathology, Mayo Clinic, Scottsdale, AZ, USA

A. Kawashima

Department of Radiology, Mayo Clinic, Rochester, MN, USA

\section{Introduction}

Solid disorders of the urethra and peri-urethral tissue are rare, and include a broad spectrum of innocuous and aggressive processes. To correctly differentiate these conditions without imaging is challenging due to non-specific and overlapping clinical signs and symptoms. Furthermore, physical examination often fails to distinguish the process origin as urethral from disorders of adjacent organs, and is unable to accurately delineate between the types of primary urethral masses $[1,2]$.

Magnetic resonance (MR) allows for high resolution multiplanar imaging of the urethra. Advancements in phased-array pelvic and endovaginal coils have led to improved evaluation of urethral and peri-urethral disorders $[1,3]$. Traditional imaging techniques, such as voiding cystourethrography and retrograde doubleballoon positive-pressure urethrography, are helpful, but are invasive and unable to characterise extraluminal spread or peri-urethral conditions [1,4]. Therefore, incorrect diagnosis and delayed treatment often occurs when relying on these modalities [1, 3]. High-resolution transvaginal, transperineal and transurethral ultrasound have been touted as reliable techniques for the diagnosis and characterisation of urethral abnormalities. While contributory when performed by an experienced sonographer, the examination can be lengthy and is operator dependent [5]. Furthermore, while ultrasonography is an important tool in the evaluation of cystic urethral and peri-urethral lesions, the contrast resolution and anatomical detail provided by MR imaging for solid lesions is unsurpassed. MR imaging can provide critical information in the management and preoperative planning of solid urethral and peri-urethral conditions.

The aim of this article is to review the normal MR anatomy of the male and female urethra, as well as the MR features of benign and malignant solid primary and secondary urethral lesions. In addition, the MR appearance of solid urethral lesion mimics will be discussed. 


\section{Normal MR anatomy of the male urethra}

The male urethra averages $17.5-20 \mathrm{~cm}$ in length, and consists of anterior and posterior portions. The posterior urethra is
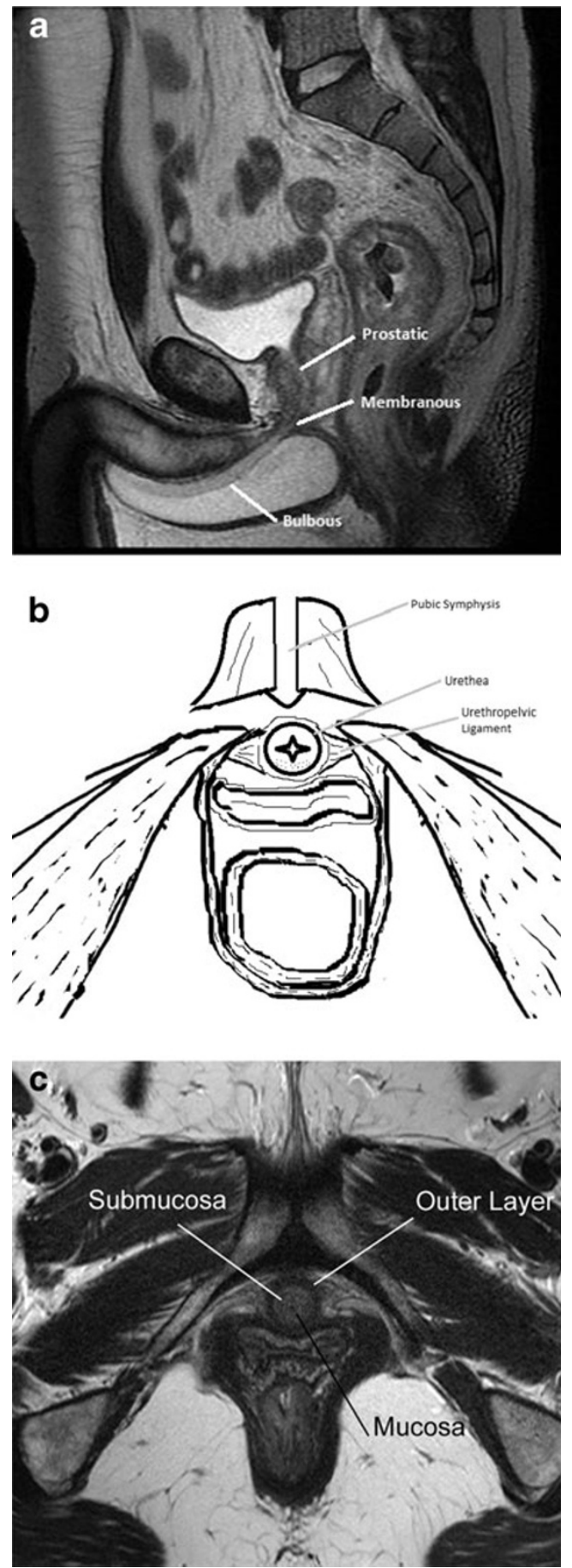

Fig. 1 a Normal anatomy. Sagittal T2-weighted image labelling the prostatic, membranous and bulbous segments of the normal male urethra. b Normal anatomy. Illustration of the normal female urethra in axial cross-section. c Normal anatomy. Axial T2-weighted image of a normal female urethra. Note the hypointense signal of the mucosa and outer muscular layer, and hyperintense submucosa subdivided into the prostatic and membranous segments [6]. T2-weighted magnetic resonance imaging (MRI) allows for visualisation of the urethral course as it transverses the prostate and urogenital diaphragm respectively [7] (Fig. 1a). The anterior portion is located in the corpus spongiosum and may be subdivided into penile and bulbous segments. The bulbous urethra extends from the inferior margin of the urogenital diaphragm to the penoscrotal junction. The penile segment then continues to the external meatus. The normal anterior urethral lumen may be difficult to delineate without a catheter in place.

While there is inter-institutional variability, the standard imaging protocol emphasises T2-weighted and gadoliniumenhanced T1-weighted sequences (Table 1). At our institution, for lesions in the anterior urethra, the patient is positioned supine, with a folded towel between the patient's legs to elevate the scrotum and penis. With the penis in the anatomical position and loosely secured in position against the midline lower abdomen, a surface coil is placed over the penis $[8$, 9]. For posterior lesions, or when obtaining pelvic images to look for inguinal or obturator lymphadenopathy, a phasedarray pelvic or body coil is used $[8,9]$. While dependent on lesion location, dynamic contrast enhanced images are most often obtained in the sagittal plane.

\section{Normal MR anatomy of the female urethra}

The female urethra averages 3-4 cm in length and extends from the bladder neck to the vestibule, forming the external meatus between the labia minora (Fig. 1b). T2-weighted MRI allows for demarcation of the concentric zonal anatomy in each of the three orthogonal planes, displayed as alternating hyper- and hypo-intense rings. Centrally, intraluminal fluid (when present) is hyperintense. The surrounding mucosa is hypointense. The

Table 1 Imaging protocols for the male and female urethra

\begin{tabular}{|c|c|c|c|c|c|c|}
\hline Sequence & $\begin{array}{l}\text { TR } \\
(\mathrm{ms})\end{array}$ & $\begin{array}{l}\mathrm{TE} \\
(\mathrm{ms})\end{array}$ & $\begin{array}{l}\text { Flip } \\
\text { angle }\end{array}$ & FOV & Matrix & $\begin{array}{l}\text { Slice } \\
\text { thickness } \\
(\mathrm{mm}) / \\
\text { spacing }\end{array}$ \\
\hline Axial T2 TSE FS & 5,400 & 102 & & 28 & $384 \times 224$ & $6 / 1$ \\
\hline $\begin{array}{l}\text { Axial high-resolution } \\
\text { T2, no FS }\end{array}$ & 4,000 & 100 & & $12-16$ & $128 \times 128$ & $3 / 1$ \\
\hline $\begin{array}{l}\text { Sagittal high-resolution } \\
\text { T2, no FS }\end{array}$ & 4,000 & 100 & & $12-16$ & $128 \times 128$ & $3 / 1$ \\
\hline $\begin{array}{l}\text { Coronal high- } \\
\text { resolution } \mathrm{T} 2 \text {, no FS }\end{array}$ & 4,000 & 100 & & $12-16$ & $128 \times 128$ & $4 / 1$ \\
\hline $\begin{array}{l}\text { Dynamic VIBE } \\
\text { pre- and post- } \\
\text { gadolinium } \\
\text { sagittal (male) } \\
\text { Axial (female) }\end{array}$ & & & 12 & 16 & $128 \times 128$ & $3 / 1$ \\
\hline $\begin{array}{l}\text { Axial VIBE post } \\
\text { gadolinium }\end{array}$ & & & 12 & $34-40$ & $320 \times 192$ & $3 / 6$ \\
\hline
\end{tabular}




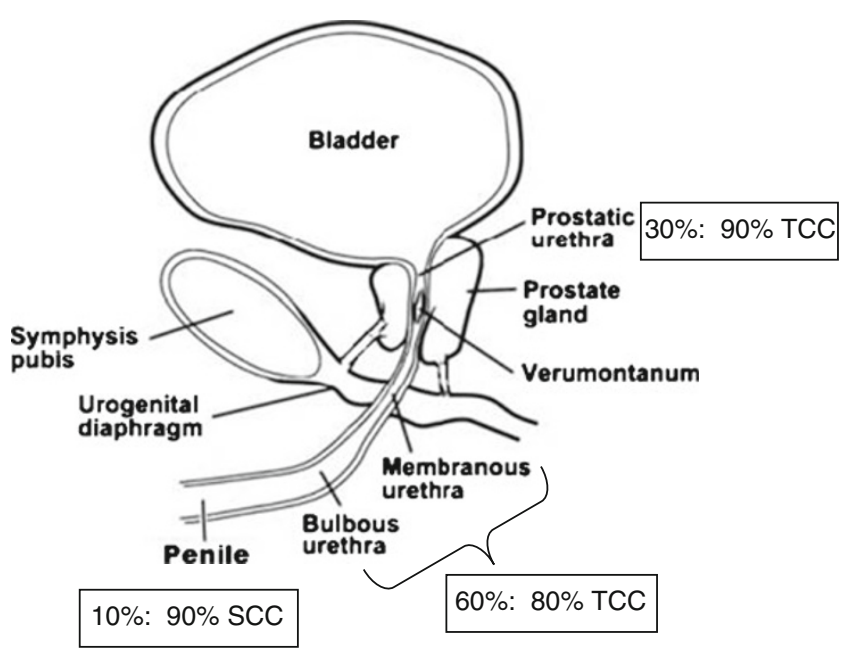

Fig. 2 Illustration of the male urethra in the sagittal plane highlighting the percentage of urethral carcinoma by location (1st percentage) and the most common histological subtype in that location (2nd percentage). TCC transitional cell carcinoma, SCC squamous cell carcinoma

submucosa, consisting of smooth muscle contiguous with the bladder neck, is hyperintense. The outermost layer, composed of circular striated muscle, is hypointense [6, 7] (Fig. 1c).

MR imaging of the female urethra is typically performed with a pelvic phased-array coil. For higher resolution imaging, endovaginal or endorectal coils are occasionally requested to delineate detailed anatomy for surgical planning. Standard protocols emphasise axial, coronal and sagittal fast spin-echo T2-weighted sequences, and axial 2D fat-suppressed spoiled gradient-echo T1-weighted sequences before and after intravenous contrast administration. In contrast to males, the dynamic phase images are most often performed in the axial plane for females.

\section{Malignant primary tumours of the male urethra}

Malignant urethral lesions in males tend to present after 50 years of age as a palpable mass in the perineum or along the length of the penile urethra. Other potential presentations include: obstructive voiding symptoms, urethral stricture or bleeding, serosanguinous discharge, perineal pain, urethral fistula, or peri-urethral abscess [6].

Urethral tumours may be categorised according to location and histology. In males, $60 \%$ arise in the bulbomembranous portion. Of these, $80 \%$ are transitional cell carcinoma (TCC), $10 \%$ are squamous cell carcinoma (SCC) and $10 \%$ are adenocarcinoma. Thirty percent arise in the prostatic urethra, of which $90 \%$ and $10 \%$ are TCC and SCC, respectively. The remaining $10 \%$ arise in the penile urethra, of which $90 \%$ are SCC and $10 \%$ are TCC [6, 10] (Fig. 2).

MRI is useful in demonstrating tumour location and size, as well as for local staging [6, 11-14] (Table 2). Relative to the normal corporal tissue, tumour will be hypointense at T1-weighted imaging (T1WI), hypointense at T2-weighted imaging (T2WI), and progressively enhance at T1WI following intravenous gadolinium-based contrast administration $(\mathrm{T} 1 \mathrm{WI}+\mathrm{C})$. As the corpora tend to be very vascular, a mass may appear to be relatively hypoenhancing. In general, stage I disease is not detectable on MRI. However, MRI readily depicts stage II tumour as invasion of the corpus spongiosum, prostate, or peri-urethral muscle (Fig. 3a). The tumour is upstaged to stage III with the presence of corpus caveronsum invasion or extension beyond the prostatic capsule (Fig. 3b and c). In addition to direct extension, urethral lesions can metastasise to regional lymph nodes. An anterior tumour spreads via inguinal lymph nodes and occasionally into the external iliac chain lymph nodes (Fig. 4). Posterior urethral tumours most commonly spread first to the deep pelvic lymph nodes, harbouring a much worse prognosis when present.

Accurate preoperative staging of urethral carcinoma is critical, as tumours sparing the corpus spongiosum and corpora cavernosa may qualify for a conservative surgical approach. In contrast, advanced lesions often require a total penectomy with creation of a perineal urethrostomy when

Table 2 Staging malignant tumours of the male and female urethra

\begin{tabular}{|c|c|c|c|}
\hline Stage & Gender & Depth of invasion & Key points \\
\hline Stage 0 and I & Either & $\begin{array}{l}\text { Non-invasive (in situ) or confined to the subepithelial } \\
\text { connective tissue }\end{array}$ & Not detectable on MRI \\
\hline \multirow[t]{2}{*}{ Stage II } & Male & $\begin{array}{l}\text { Invasion of the corpus spongiosum, prostate, } \\
\text { or peri-urethral muscle }\end{array}$ & $\begin{array}{l}\text { If present, most patients will not qualify for conservative } \\
\text { surgical management }\end{array}$ \\
\hline & Female & Invasion of the peri-urethral muscle & Loss of the normal T2WI target-zonal anatomy \\
\hline \multirow[t]{2}{*}{ Stage III } & Male & $\begin{array}{l}\text { Invasion of the corpus cavernosum and bladder neck } \\
\text { or extension beyond the prostatic capsule }\end{array}$ & \multirow[t]{2}{*}{$\begin{array}{l}\text { Also includes metastasis in a single lymph node less than } 2 \\
\mathrm{~cm} \text { (in greatest dimension), regardless of depth of invasio }\end{array}$} \\
\hline & Female & Invasion of the anterior vagina or bladder neck & \\
\hline Stage IV & Either & Invasion of other adjacent organs & $\begin{array}{l}\text { Also includes distant metastasis, metastatsis in a single node } \\
\text { greater than } 2 \mathrm{~cm} \text { (in largest dimension), or metastasis in } \\
\text { multiple nodes, regardless of depth of invasion }\end{array}$ \\
\hline
\end{tabular}


Fig. 3 a Malignant male tumour: stage II bulbar urethral squamous cell carcinoma. The patient presented with a palpable non-tender mass. Sagittal T2-weighted image demonstrates a bulbar urethral mass invading the corpus spongiosum (arrows). b Malignant male tumour: stage III penile urethral squamous cell carcinoma. In a different patient, sagittal T2weighted image demonstrates a large penile urethral mass involving the corpus sponsiosum (arrows) and focally invading the tunica albuginea and corpora cavernosa (block arrow). c Malignant male tumour: penile urethral squamous cell carcinoma. Retrograde urethrogram of the same patient as b demonstrates irregular narrowing of the penile urethra secondary to the mass (arrows). The full extent of the mass and the corpora cavernosa invasion is not appreciated by this modality

involving the anterior urethra and anterior exenteration with radical cystoprostatourethrectomy and urinary diversion when involving the posterior urethra $[15,16]$.

\section{Malignant primary tumours of the female urethra}

While rare in both genders, urethral malignancies are more common in females, with a female-to-male ratio of 4 to 1 [6, 17]. As with males, the presenting population tends to be greater than 50 years old. Potential associated symptoms include: urethral bleeding, urinary frequency, obstructive symptoms, palpable urethral mass or induration.

Female urethral malignancies are classified as anterior, where tumour is isolated to the distal third of the urethra, or entire, where the proximal two-thirds of the urethra $+/-$ the anterior segment are involved (Fig. 5). There is no distinction in treatment between tumours involving the entire urethra and those isolated to the proximal two-thirds. The anterior subtype accounts for approximately $45 \%$ of urethral tumours, and tend to be less extensive and low grade [7]. Histological subtypes are $60 \%$ SCC, $20 \%$ TCC, $10 \%$ adenocarcinoma, $8 \%$ undifferentiated tumour and sarcoma, and $2 \%$ melanoma [6].

MRI can play an important role in local staging (Table 2). Tumours of squamous cell or transitional cell origin will be relatively hypointense on T1WI and hypointense to intermediate intensity at T2WI and demonstrate heterogeneous enhancement. In contrast, adenocarcinoma tends to be relatively $\mathrm{T} 2$ hyperintense, with variable enhancement [5]. As with urethral tumours in males, MRI has limited utility in depicting stage I disease. However, the target-like appearance of the normal urethra on axial T2-weighted images will be disrupted in stage II lesions [6, 18]. With stage III and IV disease, differentiation of primary urethral lesions from those of the vulva or vagina may be difficult (Fig. 6).

\section{Benign urethral lesions}

Solid benign urethral lesions are very rare. The more commonly encountered include: leiomyomas, haemangiomas
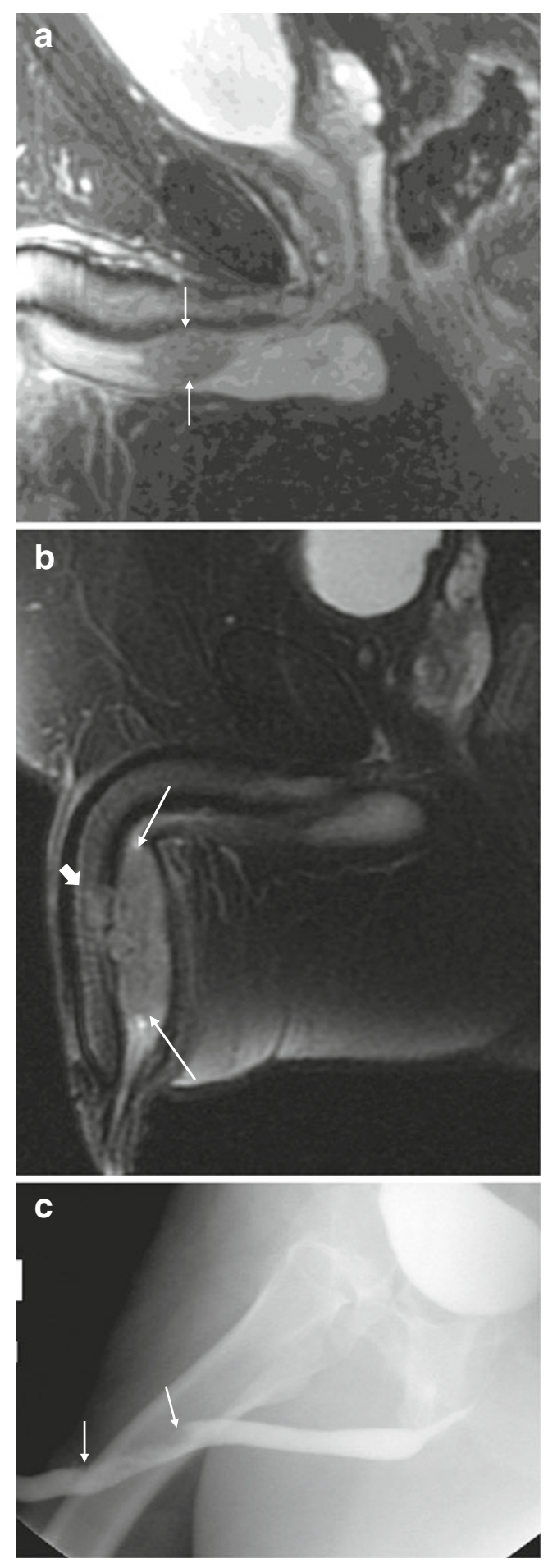

and fibroepithelial polyps [6, 7, 12, 19, 20]. While MR imaging characteristics of benign lesions overlap with those of malignant tumours, there are imaging characteristics which may assist in the diagnosis.

Urethral leiomyomas originate from smooth muscle fibres of the urethra. They tend to present in reproductive age females as difficulty in micturition and urinary 

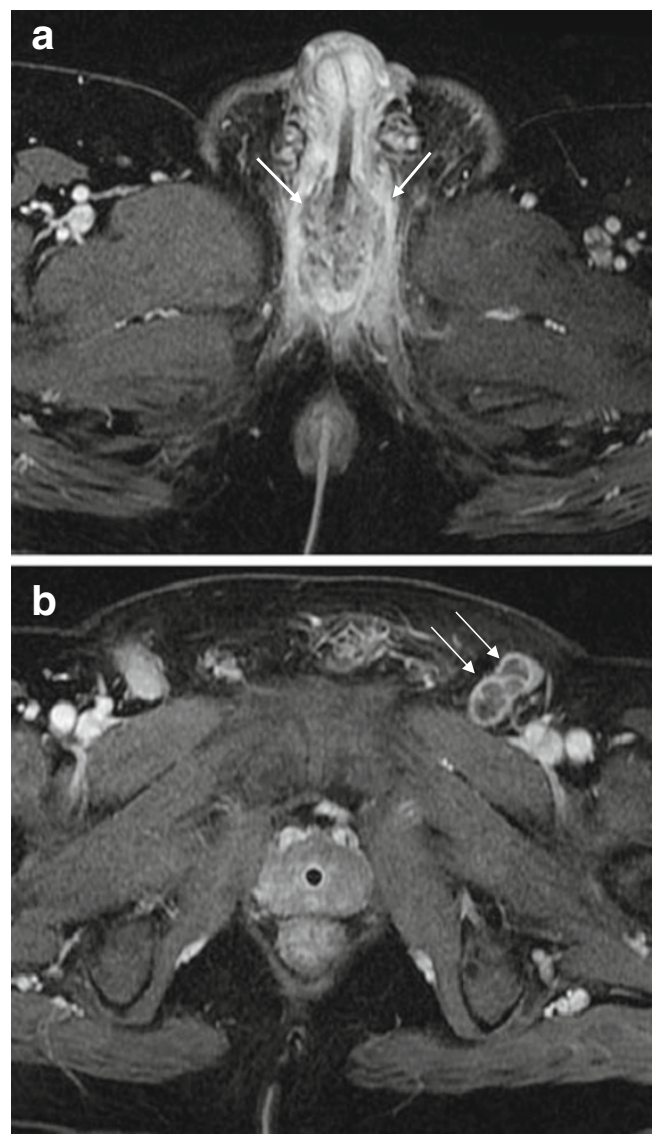

Fig. 4 a Malignant male tumour: urethral squamous cell carcinoma with lymph node metastases. Axial T1-weighted image after intravenous gadolinium-based contrast administration demonstrates a heterogeneously enhancing bulbomembranous squamous cell carcinoma (arrows). b Malignant male tumour: urethral squamous cell carcinoma with lymph node metastases. Axial T1-weighted image after intravenous gadolinium-based contrast administration through the pelvis in the same patient demonstrates necrotic left inguinal lymphadenopathy (arrows). The presence of multiple $(>1)$ regional lymph node metastases upstages to stage IV

tract infections, dyspareunia, and/or haematuria [19, 20]. At MRI, urethral leiomyomas tend to be well demarcated, and follow signal characteristics of a non-degenerated uterine leiomyoma; low to intermediate signal intensity on T1WI, low to intermediate signal intensity on T2WI and fairly homogeneous enhancement (Fig. 7).

Haemangiomas of the urethra are believed to arise from embryonic remnants of angioblastic cells that failed to develop into normal blood vessels [21]. Urethral haemangiomas occur much more commonly in males, with haematuria and/or haematospermia being the presenting symptoms [22, 23]. At MRI, high T2WI signal similar to the characteristic appearance of a hepatic haemangioma has been reported [21]. Ultimately, cystourethroscopy and biopsy are required for diagnosis [21-23].
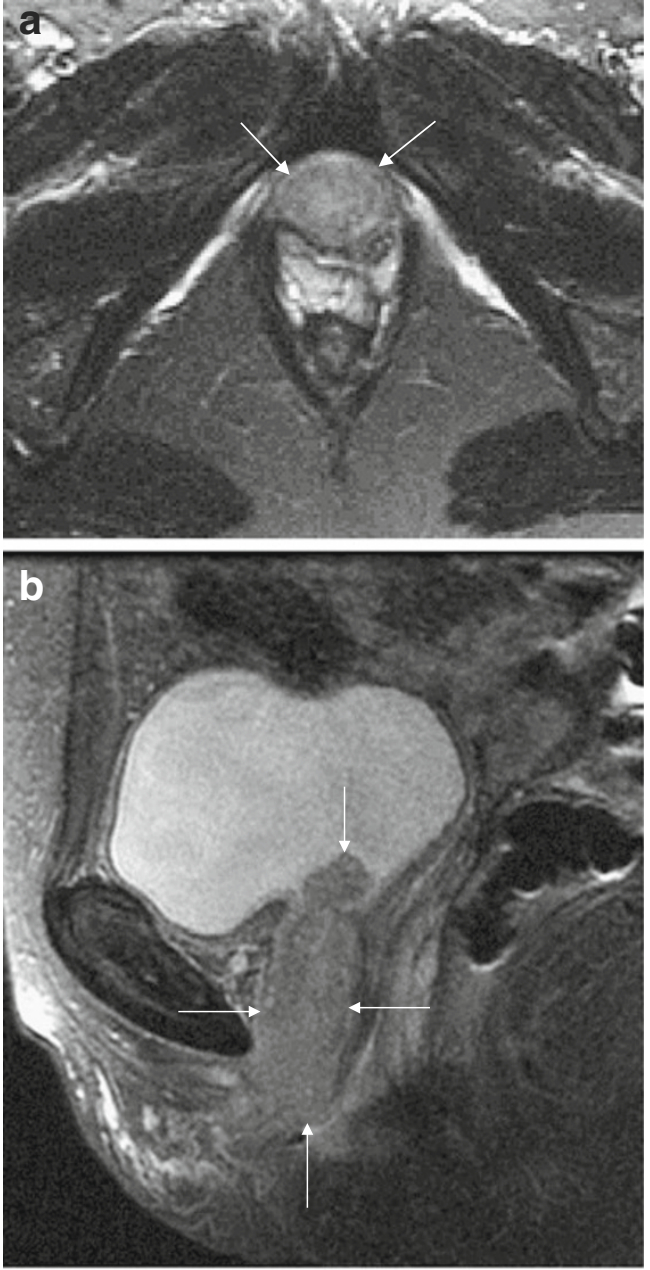

Fig. 5 a Malignant female tumour: 'entire' urethral squamous cell carcinoma. Axial T2-weighted image demonstrates an infiltrative mass replacing the normal concentric appearance of the female urethra (arrows). b Malignant female tumour: 'entire' urethral squamous cell carcinoma. Sagittal T2-weighted image shows the mass involving the entire urethra (arrows), extending into the bladder (top arrow)

Fibroepithelial polyps of the urethra present more commonly in children [24]. When presenting in adulthood, the clinical, radiographic and endoscopic findings will most often be that of an obstructing mass at the bladder neck and prostatic urethra [24-26]. MRI can demonstrate the typical polypoid shape, with a stalk connected to the bladder neck. The tumour contains abundant stroma; therefore, homogeneous enhancement after intravenous gadolinium administration is characteristic [27].

\section{Secondary tumours to the urethra}

Secondary tumours involving the urethra most commonly occur by direct invasion or contiguous spread from 

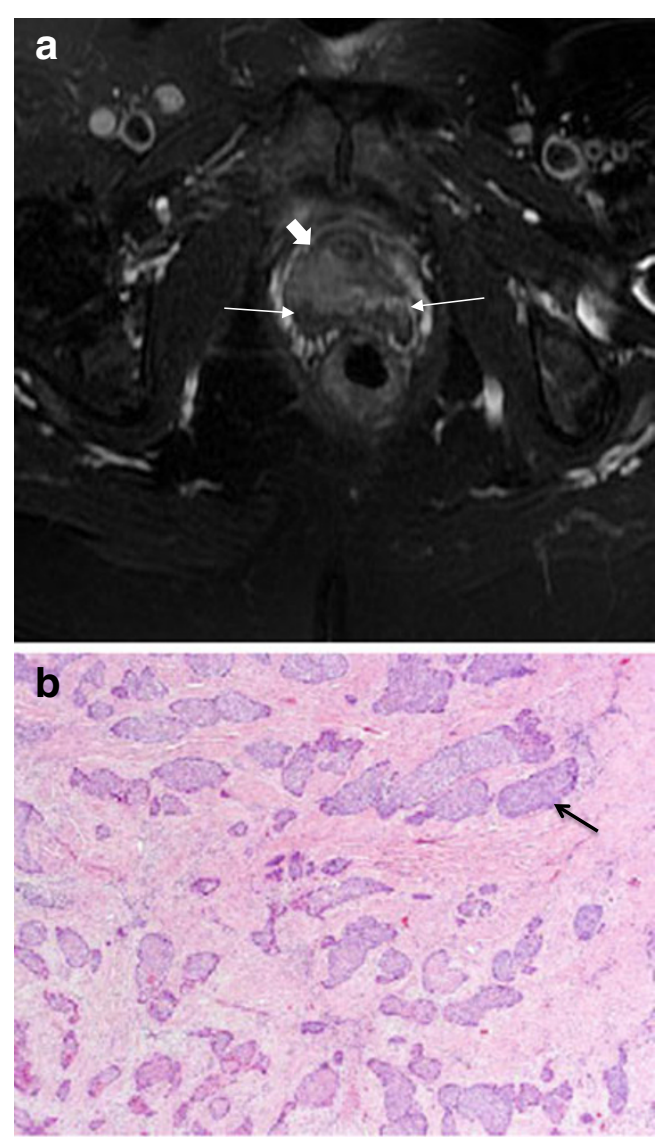

Fig. 6 a Malignant female tumour: invasive squamous cell carcinoma. Axial T2-weighted image demonstrates disruption of the target sign by a mass (block arrow), which extends posteriorly to invade the anterior wall of the vagina (arrows). b Malignant female tumour: invasive squamous cell carcinoma. Histological specimen shows infiltrating islands of malignant cells (purple) throughout the image, representing squamous cell carcinoma of the urethra. The arrow points to one of these islands. (Haematoxylin and eosin, original magnification $40 \times$ )

adjacent organs. This includes primary tumours originating in the prostate, rectum, spermatic cord, penis, vagina (Fig. 8), vulva or bladder. Primary TCC of the urinary bladder has been reported to involve the proximal urethra in $2-13 \%$ of women at the time of diagnosis $[1,3]$.

Bladder TCC may also spread to the anterior urethra by seeding during urethral instrumentation or at cystectomy, and will be difficult to differentiate from metachronous urethral TCC both clinically and radiographically. This has been described at urethrography as multiple small mucosal nodules $[6,12]$. A similar appearance can be demonstrated at MRI, with multiple small enhancing mucosal nodules (Fig. 9).

Haematogenous metastases to the corpus spongiosum are occasionally seen with melanoma, prostate, bladder, colon, testicular and renal primaries [6, 12] (Fig. 10). Erosion into the urethra will then produce urethral irregularities.
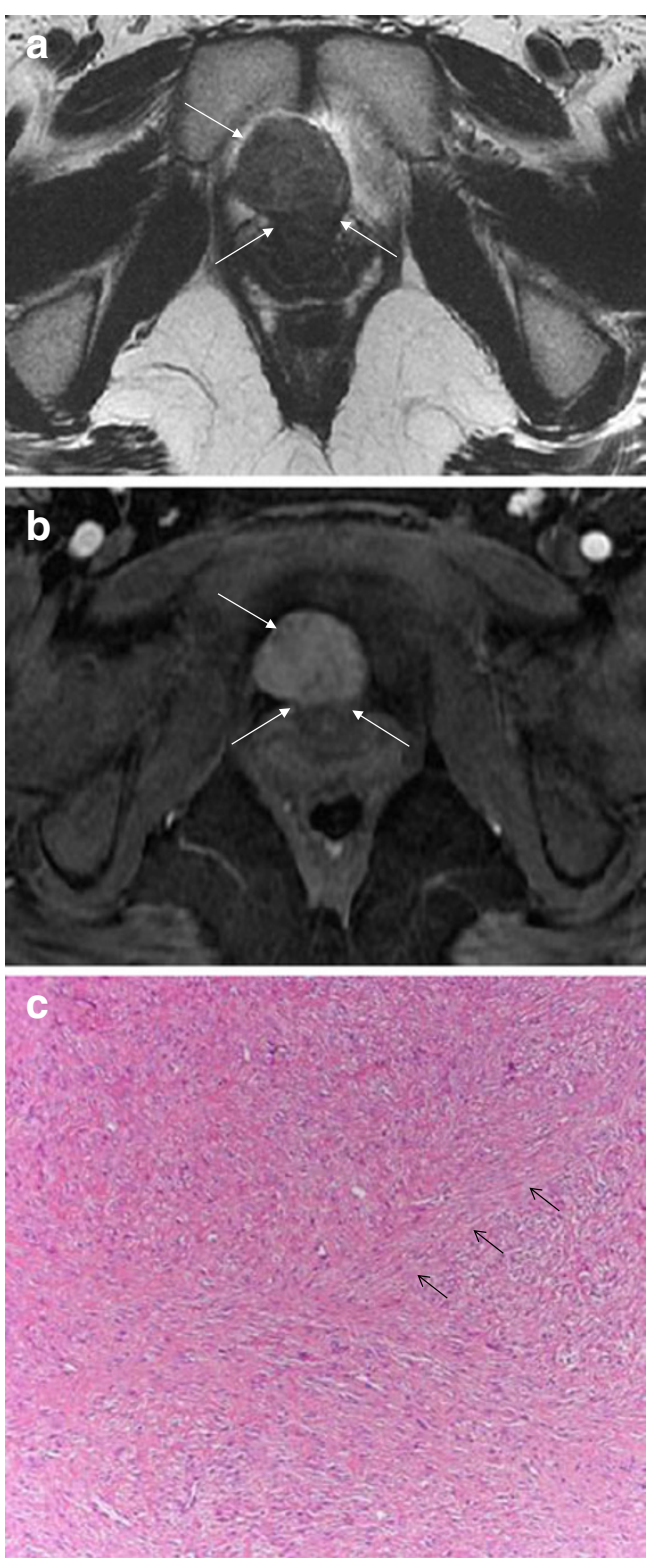

Fig. 7 a Benign lesions: urethral leiomyoma. Axial T2-weighted image shows an exophytic, well demarcated mass arising from the urethra anteriorly (arrows). b Benign lesions: urethral leiomyoma. Axial T1weighted image after intravenous gadolinium-based contrast administration demonstrates homogeneous enhancement of the mass (arrows). c Benign lesions: urethral leiomyoma. Histological specimen shows characteristic intersecting fascicles of smooth muscle throughout the image in this benign stromal tumour. The arrows point to one of these fascicle bands. (Haematoxylin and eosin, original magnification $100 \times$ )

\section{Urethral tumour mimics}

Complex cystic peri-urethral lesions may mimic a solid mass on imaging. Examples include inflamed/infected urethral diverticula and urethral bulking agents. An uncomplicated urethral diverticulum will be high signal on T2WI and demonstrate little, if any, enhancement (Fig. 11a). In a 

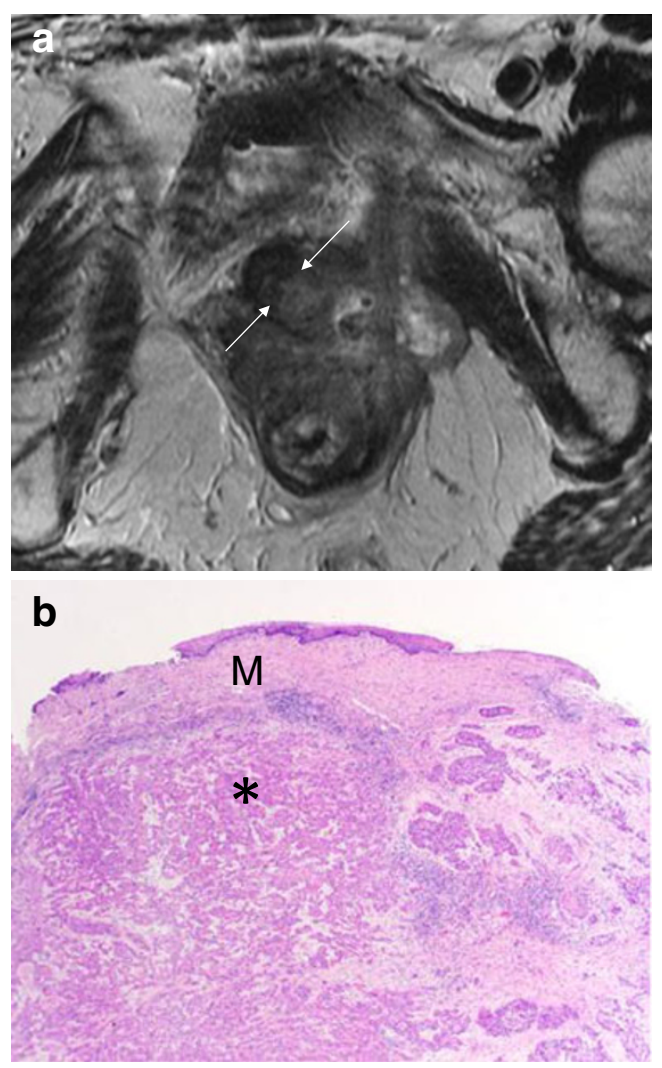

Fig. 8 a Secondary tumours involving the urethra: direct extension. Vagina squamous cell carcinoma invading the urethra. Axial T2weighted image shows an infiltrative mass centred at the vagina, extending anteriorly to invade the posterior-left wall of the urethra (arrows). b Secondary tumours involving the urethra: direct extension. Histological specimen shows the squamous cell carcinoma $(*)$ undermining the normal vaginal squamous mucosa $(M)$ above. (Haematoxylin and eosin, original magnification $40 \times$ )

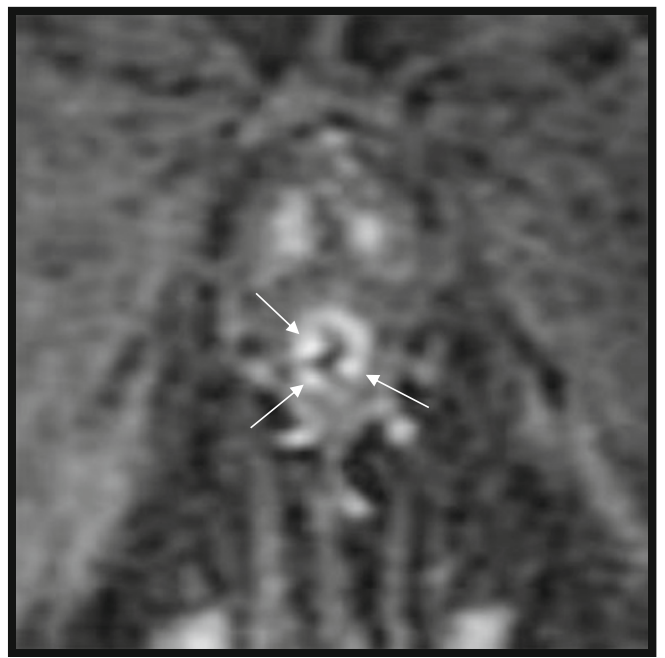

Fig. 9 Secondary tumours to the urethra: seeding of the urethra after radical cystoprostatectomy for transitional cell carcinoma. Zoomed in coronal T1-weighted image after intravenous gadolinium-based contrast administration demonstrates multiple small enhancing mucosal nodules within the urethra (arrows)
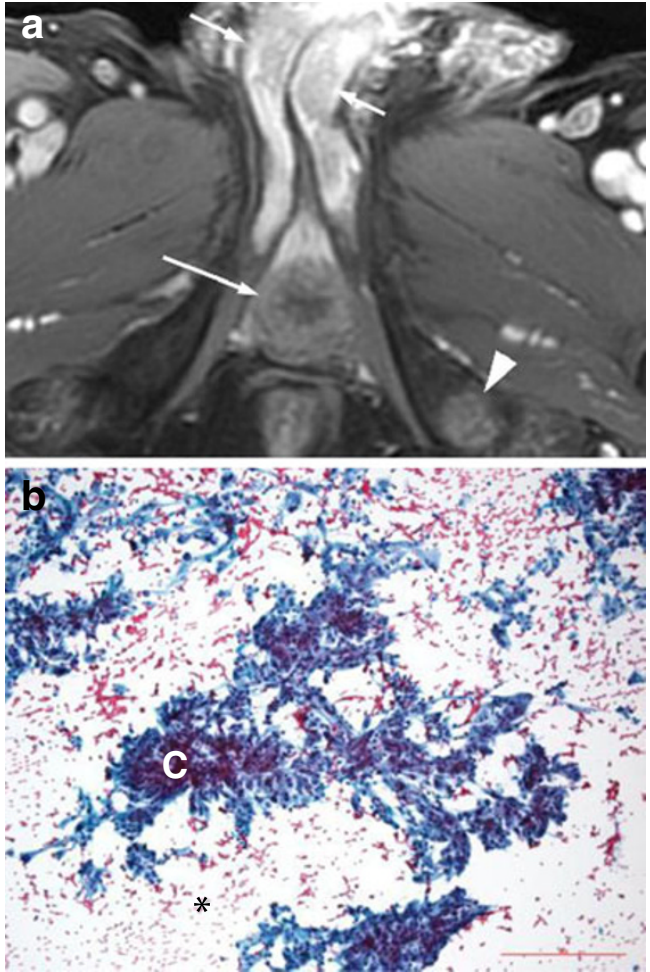

Fig. 10 a Secondary tumours to the corpora: renal cell carcinoma metastasis. A 74-year-old man with metastatic renal cell carcinoma presented with hesitation and difficulty in urination as well as a palpable firm mass in the right base of the penis. Axial T1-weighted image after intravenous gadolinium-based contrast administration shows enhancing masses in the bilateral corpora cavernosa (short arrows) and corpus spongiosum (long arrow) as well as in the left ischial tuberosity (arrowhead). The corporal lesions are hypoenhancing relative to adjacent normal corporal tissue however hyperenhancing relative to the nearby musculature. b Secondary tumours to the corpora: renal cell carcinoma metastasis. Histological specimen using Papanicolaou stain shows metastatic carcinoma with clear cell features, consistent with metastatic grade 3 (of 4) renal cell carcinoma. There are clusters of poorly cohesive cells (C) with low nucleo-cytoplasmic ratio, abundant pale cytoplasm and pale, vacuolated background $(*)$

complex urethral diverticulum, the fluid is intermediate signal on T2WI (Fig. 11b), and may enhance heterogeneously.

The MR characteristics of urethral bulking agents vary based on their composition and the time since injection. The most commonly used agents are collagen suspension or coated microbeads. Recently injected peri-urethral collagen suspension is intermediate to hyperintense on T2WI and lacks enhancement (Fig. 12). The collagen suspension becomes isointense to hypointense with time on T1WI and T2WI. Coated microbeads are hypointense on T2WI and T1WI and lack enhancement, regardless of age [28].

Amyloidosis is often a systemic condition, however, can be a diagnostic dilemma when localised to the urethra. The clinical presentation and imaging features overlap with that of malignancy. At MRI, amyloid depositions are hypointense on T2WI (Fig. 13a) and hypointense on T1WI, with avid enhancement [29, 30]. 

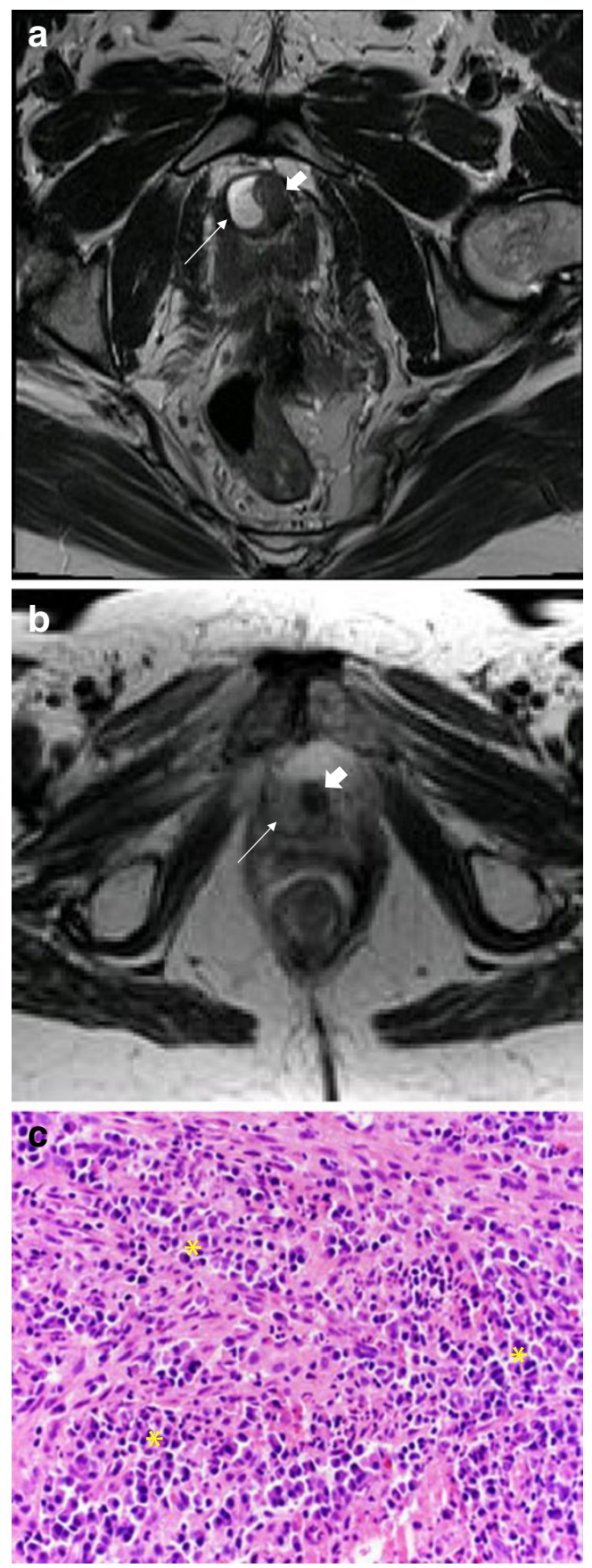

Fig. 11 a Urethral tumour mimics: Complex/inflamed diverticulum mimicking a mass. Axial T2-weighted image shows a high signal collection (arrow) along the right margin of the urethra (block arrow), in keeping with an uncomplicated urethral diverticulum. b Urethral tumour mimics: complex/inflamed diverticulum mimicking a mass. In contrast, axial $\mathrm{T} 2$-weighted image in a different patient demonstrates an inflamed diverticulum as intermediate signal (arrow) along the right margin of the urethra (block arrow). c Urethral tumour mimics: complex/inflamed diverticulum mimicking a mass. Histological section shows an intense acute and chronic inflammatory infiltrate throughout the image $(*)$, with a mix of neutrophils, lymphocytes and monocytes. (Haematoxylin and eosin, original magnification $400 \times$ )
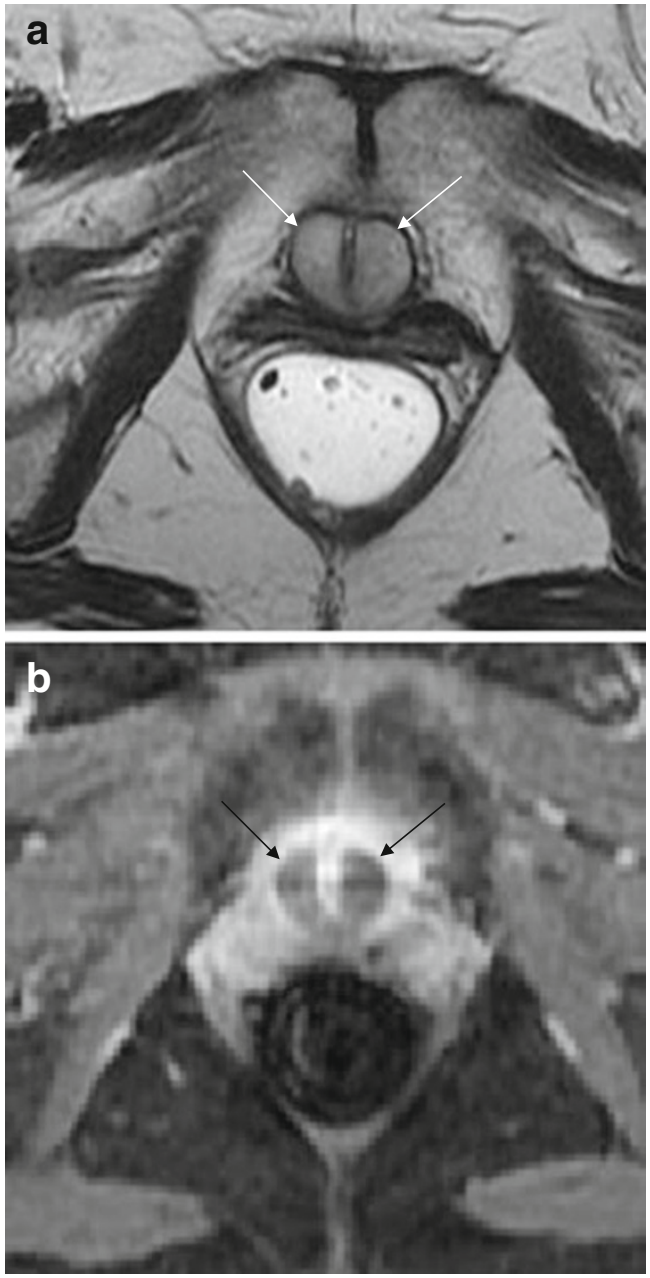

Fig. 12 a Urethral tumour mimics: urethral bulking agents. Axial T2weighted image demonstrates recently injected peri-urethral collagen suspension to be relatively hyperintense. b Urethral tumour mimics: urethral bulking agents. With T1-weighted image after intravenous gadolinium-based contrast administration, recently injected peri-urethral collagen suspension lacks enhancement (arrows)

Penile ultrasonography plays a complimentary role, depicting intramural calcifications as echogenic foci with posterior shadowing [30] (Fig. 13b).

\section{Conclusion}

MRI is an important imaging technique in the evaluation of the spectrum of solid urethral and peri-urethral lesions in adults. There is high accuracy in local staging of primary urethral carcinomas in both males and females due to excellent soft tissue resolution. Disruption of the "target-like" zonal anatomy of the female urethral wall indicates peri-urethral extension. It is important to be aware of benign urethral lesions, particularly those that may mimic solid urethral masses. 

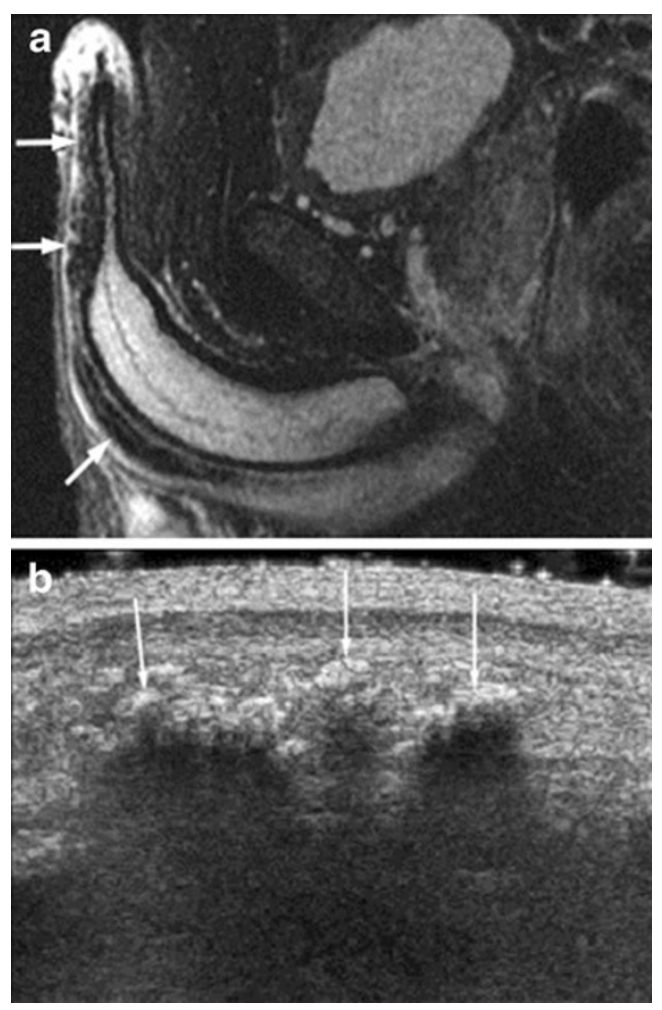

Fig. 13 a Urethral tumour mimics: primary urethral amyloidosis. Sagittal T2-weighted image shows narrowing of the penile urethra by multiple hypointense amyloid deposits (arrows). Reprinted with permission from Kawashima et al. [30]. b Urethral tumour mimics: primary urethral amyloidosis. Correlative ultrasound demonstrates intramural calcifications. Reprinted with permission from Kawashima et al. [30]

\section{Conflict of interest None.}

Open Access This article is distributed under the terms of the Creative Commons Attribution License which permits any use, distribution, and reproduction in any medium, provided the original author(s) and the source are credited.

\section{References}

1. Chaudhari VV, Patel MK, Douek M, Raman SS (2010) MR imaging and US of female urethral and periurethral disease. Radiographics 30(7):1857-1874

2. Handel LN, Leach GE (2008) Current evaluation and management of female urethral diverticula. Curr Urol Rep 9(5):383-388

3. Rufford J, Cardozo L (2004) Urethral diverticula: a diagnostic dilemma. BJU Int 94(7):1044-1047

4. Hahn WY, Israel GM, Lee VS (2004) MRI of female urethral and periurethral disorders. AJR Am J Roentgenol 182(3):677-682

5. Prasad SR, Menias CO, Narra VR, Middleton WD, Mukundan G, Samadi N, Heiken JP, Siegel CL (2005) Cross-sectional imaging of the female urethra: technique and results. Radiographics 25(3):749-761

6. Kawashima A, Sandler CM, Wasserman NF, Leroy AJ, King BF Jr, Goldman SM (2004) Imaging of urethral disease: a pictorial review. Radiographics 24:S195-S216
7. Ryu F, Kim B (2001) MR imaging of the male and female urethra. Radiographics 21:1169-1185

8. Pretorius ES, Siegelman ES, Ramchandani P, Banner MP (2001) MR imaging of the penis. Radiographics 21 Spec No:S283-S298, discussion S298-9

9. Singh AK, Saokar A, Hahn PF, Harisinghani MG (2005) Imaging of penile neoplasms. Radiographics 25(6):1629-1638

10. Donat SM, Cozzi PJ, Herr HW (2002) Surgery of penile and urethral carcinoma. In: Walsh PC, Reitik AB, Vaughan ED Jr, Wein AJ (eds) Campbell's urology, 8th edn. Saunders, Philadelphia, pp 2983-2999

11. Hricak H, Marotti M, Gilbert TJ et al (1988) Normal penile anatomy and abnormal penile conditions: evaluation with MR imaging. Radiology 169:683-690

12. Wasserman NF (2000) Urethral neoplasms. In: Pollack HM, McClennon BL (eds) Clinical urography, 2nd edn. Saunders, Philadelphia, pp 1699-1715

13. Vapnek JM, Hricak H, Carroll PR (1992) Recent advances in imaging studies for staging of penile and urethral carcinoma. Urol Clin North Am 19:257-266

14. Shirkhoda A, Salmanzadeh A, Jafri SZ, Konez O, Spencer W (2000) Urethral leiomyosarcoma: evaluation by MRI with pathologic correlation. J Comput Assist Tomogr 24:423-425

15. Lynch DF Jr (2003) Carcinoma of the urethra. In: Kufe DW, Pollock RE, Weichselbaum RR et al (eds) Holland-Frei cancer medicine, 6th edn. BC Decker, Hamilton

16. Bissada NK, Yakout HH, Fahmy WE, Gayed MS, Touijer AK, Greene GF, Hanash KA (2003) Multi-institutional long-term experience with conservative surgery for invasive penile carcinoma. $\mathrm{J}$ Urol 169(2):500-502

17. Kageyama S, Ueda T, Kushima R, Sakamoto T (1997) Primary adenosquamous cell carcinoma of the male distal urethra: magnetic resonance imaging using a circular surface coil. J Urol 158:1913-1914

18. Morikawa K, Togashi K, Minami S et al (1995) MR and CT appearance of urethral clear cell adenocarcinoma in a woman. $\mathrm{J}$ Comput Assist Tomogr 19:1001-1003

19. Fasih N, Prasad Shanbhogue AK, Macdonald DB, Fraser-Hill MA, Papadatos D, Kielar AZ et al (2008) Leiomyomas beyond the uterus: unusual locations, rare manifestations. Radiographics 28:1931-1948

20. Lee MC, Lee SD, Kuo HT, Huang TW (1995) Obstructive leiomyoma of the female urethra: report of a case. J Urol 153(2):420-421

21. Uchida K, Fukuta F, Ando M, Miiyake M (2001) Female urethral hemangioma. J Urol 166(3):1008

22. Rao AR, Motiwala H (2005) Urethral hemangioma. Urology 65(5): 1000

23. Saito S (2008) Posterior urethral hemangioma: one of the unknown causes of hematuria and/or hematospermia. Urology 71(1):168.e11168.e14

24. Aita GA, Begliomini H, Mattos D Jr (2005) Fibroepithelial polyp of the urethra. Int Braz J Urol 31:155-156

25. Salehi M, Falahatkar S, Neiroomand H, Akbarpour M (2009) Fibroepithelial congenital polyp of prostatic urethra in an adult man. Urol J 6:301-302

26. Williams TR, Wagner BJ, Corse WR, Vestevich JC (2002) Fibroepithelial polyps of the urinary tract. Abdom Imaging 27:217-221

27. Li H, Sugimura K, Boku M, Kaji Y, Tachibana M, Kamidono S (2003) MR findings of prostatic urethral polyp in an adult. Eur Radiol 13:L105-L108

28. Bridges MD et al (2005) Urethral bulking agents, imaging review. AJR Am J Roentgenol 185:257-264

29. Ichioka K, Utsunomiya N, Ueda N, Matsui Y, Yoshimura K, Terai A (2004) Primary localized amyloidosis of urethra: magnetic resonance imaging findings. J Urol 64(2):376-378

30. Kawashima A, Alleman WG, Takahashi N, Kim B, King BF Jr, LeRoy AJ (2011) Imaging evaluation of amyloidosis of the urinary tract and retroperitoneum. Radiographics 31(6):1569-1582 